\title{
Cell transfection-a short approach of DNA mutagenesis
}

\begin{abstract}
The transfection method could be used for a variety of applications: large scale production of proteins of biological interest, inserting genes into eukaryotic cells to these acquires desired characteristics; analyze maintenance of genes and their expression; and future application in vivo as vaccines DNA and gene therapy. In order to improve and establish new conditions, as well as verify the most current techniques of cell transfection, this review aims to bring together the most current methods in order to be applied to the study and development of new techniques, always looking for the improving from limitations of existing techniques, afterward to be choose more appropriate methods for the required goals.
\end{abstract}

Keywords: cell transfection, virus transfection, DNA, transfection
Volume 3 Issue 2 - 2017

Thaís H Theizen,' Adriana De Melo, I,2 Daisy Machado, ${ }^{1,2}$ Marcelo Lancellotti ${ }^{1,2}$

'Laboratório de Biotecnologia, Brazil

${ }^{2}$ Faculdade de Ciências Farmacêuticas, Brazil

Correspondence: Marcelo Lancellotti, Labiotec, Instituto de Biologia, Bloco F, Unicamp, Cidade Universitária Zeferino Vaz, Barão Geraldo, Campinas, SP, Brazil, 1347। -083, Tel +55 19 $35216150, \mathrm{Fax}+551935216129$,

Email marcelo.lancellotti@fcf.unicamp.br

Received: October 17,2016 | Published: May 26, 2017
Abbreviations: HSV, herpes simplex virus; MLV, moloney leukemia virus; GFP, green fluorescent protein; PEI, polyethylenimine; RLU, relative light units

\section{Introduction}

The transfection methods could be classified as transient or stable transfection and transfection in vitro or in vivo. Transient transfection is when DNA is introduced into the nucleus but is not incorporated into the chromosome; or permanent when the DNA is incorporated into the chromosome or maintained as an episome. ${ }^{1}$ In vitro Transfection is the introduction of DNA into cultured cells. The cell culture is conducted in culture flasks and their maintenance, conditions $(\mathrm{pH}$, nutrients, temperature) can be controlled by the manipulator. In the in vivo transfection DNA is introduced into the body of the target cells of higher living beings. Assay-based reporter technology, together with the availability of transfection reagents, provides the foundation to study mammalian promoter and enhancer sequences, transacting proteins such as transcription factors, mRNA processing, protein interactions, translation and recombination events. ${ }^{2,3}$ Transfection is a method that neutralizes or obviates the issue of introducing negatively charged molecules (e.g., phosphate backbones of DNA and RNA) into cells with a negatively charged membrane. Physical methods like microinjection or electroporation simply punch through the membrane and introduce DNA directly into the cytoplasm.

\section{Biological methods}

\section{Viral methods}

While transfection has been used successful for gene transfer, the use of viruses as vectors has been explored as an alternative method to deliver foreign genes into cells and as a possible in vivo option. Adenoviral vectors are useful for gene transfer due to several key features:

i. They rapidly infect a wide scope of human cells and can achieve high degrees of gene transfer compared to other available vectors ii. Adenoviral vectors can accommodate relatively large segments of DNA (up to $7.5 \mathrm{~Kb}$ ) and transduce these transgenes in nonproliferating cells

iii. Adenoviral vectors are relatively easy to manipulate using recombinant DNA techniques. ${ }^{4,5}$ Other vectors of interest include adeno-associated virus, Herpes Simplex Virus (HSV), retroviruses and lentiviruses, a subset of the retrovirus family.

Also, lentiviruses (e.g., HIV-1) are of particular interest because they are well studied, can infect quiescent cells and can integrate into the host cell genome to allow stable, long-term transgene expression. ${ }^{6}$ For adenoviral vectors, packaging capacity is low and production is labor-intensive. ${ }^{7}$ With retroviral vectors, there is the potential for activation of latent disease and, if there are replication-competent viruses present, activation of endogenous retroviruses and limited transgene expression., ${ }^{4,5}$

\section{Retroviruses}

Retroviruses are vectors that are characterized by continuous and stable expression due to the integration of viral genome into the host cell chromosome. ${ }^{8,9}$ The Moloney leukemia virus (MLV) has been the most widely used in clinical tests. They consist of two genes (rep and $c a p$ ), but require additional genes to replicate and generally used the adenovirus or herpes simplex virus. ${ }^{10}$ However, these vectors can package DNA sizes small (3-4.0 Kb). ${ }^{11}$ In addition, the HSV has emerged as an attractive vector for gene therapy, especially diseases related to the nervous system. Thus, chemical methods were initially developed based on the use of positive charge carriers that interact with DNA, neutralize its charge and forming a complex that enables its passage through the membrane. Diethylaminoethyl DEAE-dextran is a cationic polymer that tightly associates with negatively charged nucleic acids. However, this technique is not generally useful for stability or long-term transfection studies that rely upon the integration of transferring DNA into the chromosome. ${ }^{12}$ Other synthetic cationic polymers have been used to transfer DNA into cells, including polybrene, ${ }^{13}$ polyethyleneimine and dendrimers. ${ }^{13-15}$ 


\section{DNA co-precipitation with calcium phosphate}

Calcium phosphate co-precipitation became a popular transfection technique following the systematic examination of this method in the early $1970 \mathrm{~s}^{16,17}$ The authors examined the performance of several cations and effects of their concentration on transfection. Calcium phosphate co-precipitation is widely used because the components are easily available, inexpensive and effective in many different types of cultured cells. The calcium phosphate transfection is routinely used for both transient and stable transfection of a variety of cell types. In addition, calcium phosphate appears to provide protection against intracellular and serum nucleases. ${ }^{18,19}$ Also, small $\mathrm{pH}$ changes $( \pm 0.1)$ can compromise the efficacy of calcium phosphate transfection. ${ }^{20}$

Liposomes formed by cationic lipids: The term "liposome" refers to lipid bi-layers that forms colloidal particles in an aqueous medium. ${ }^{21,22}$ Artificial liposomes were being used to deliver DNA into cells, ${ }^{23}$ the advances in liposomal vehicles were development of synthetic cationic lipids by Felgner et al. ${ }^{24}$ Liposome-mediated delivery offers advantages such as relatively high efficiency of gene transfers, ability to transfect certain cell types that are resistant to calcium phosphate or DEAE-dextran, in vitro and in vivo applications, successful delivery of DNA of all sizes of oligonucleotides at yeast artificial chromosomes, ${ }^{24-30}$ delivery RNA ${ }^{31,32}$ and protein..$^{33}$ While DNA will need to enter the nucleus, the cytoplasm is the site of action for RNA, protein or antisense oligonucleotides delivered via liposomes. Also the liposomes formed by anionic lipids, which are negatively charged liposomes have a great fusion cell. ${ }^{21}$ They used a mixture of phosphatidylcholine and phosphatidylglycerol, lipids that are present in egg yolk, to prepare negatively charged liposomes.

Liposomes formed by cationic cholesterol derivatives: Cationic Liposomes are composed of three distinct parts: cholesterol structure, an end to an amino group and a binding region between the two previous structures. This derivative was made by DC-col and presented good transfection efficiency and cytotoxicity. ${ }^{22}$ Direct microinjection into cultured cells or nuclei is an effective, although the laborious technique to deliver nucleic acids into cells by means of a fine needle. This method has been used to transfer DNA into embryonic stem cells that are used to produce transgenic organisms and to introduce antisense RNA into C. elegans. However, the apparatus is costly and the technique extremely labor-intensive, thus it is not an appropriate method for studies that require a large number of transfected cells.

Because, the apparatus is costly and the technique is extremely laborintensive, it is not an appropriate method for studies that require a large number of transfected cells. This technique is often used for cell types such as plant protoplasts, which are difficult to transfect by other methods. The technique requires fine-tuning and optimization of pulse duration and strength of each type of cell used. In addition, electroporation often requires more cells than chemical methods because of substantial cell death and extensive optimization often is required to balance transfection efficiency and cell viability. More modern instrumentation allows nucleic acid delivery to the nucleus and successful transfer of DNA and RNA to primary and stem cells. Episomal vectors are usually transported into cells via electroporation, although many problems electroporation derivatives led to the search for alternative transfection protocols, such as the use of transfection reagents that are inexpensive and easy to handle. ${ }^{25,26}$ Biolistic particle delivery is relatively costly for many research applications, but the technology also can be used for genetic vaccination and agricultural applications. The transfection method that will be used will depend on the experiment to be performed, the study will be short or long term and the reagent used, types of transfected molecules. Several liposomal transfection reagents do not have wide application in different cell types with low toxicity, which use the polycationic head group attached to a lipid structure as a cover helping to deliver nucleic acids into eukaryotic cells. But also in non-dividing cells, research has shown that Lipofectamine improves the efficiency of transfection, which suggests that it additionally helps the transfected genetic material penetrate the intact nuclear envelope.

\section{Current methods used for cell transfection}

Several gene delivery systems have been developed to introduce genes of interest into cells. Virus- mediated gene delivery systems are often preferred because of its high transfection efficiency and simple mechanism infection. Lipofectamine reagent contains lipid subunits that can form liposomes in an aqueous environment, is a cationic liposome formulation, which complexes with negatively charged nucleic acid molecules, which allow them to overcome the electrostatic repulsion of the cell membrane. The DNA-containing liposomes (with a positive charge on their surfaces) can fuse with the negatively charged plasma membrane of living cells, due to the neutral co-lipid mediating fusion of the liposome with the cell membrane, allowing nucleic acid to cross into the cytoplasm and contents to be available to the cell for replication or expression. The liposome containing the DNA binds the endosomal membrane due to the neutral co- lipid, allowing the nucleic acid to pass through the cytoplasm and the DNA content becomes available to the cell to express and replication of a certain gene. In vitro gene assays typically use plasmids expressing luciferase or green fluorescent protein (GFP) as reporter genes, which are most often performed in either 6-or 96-well formats. ${ }^{27}$ Magnetic nanoparticles of iron oxide combined with cationic polymers (PEI) conjugated to a fluorophore label can be detected by fluorescence microscopy are being used in transfection of genetic material (pDNA and siRNA) to stem cells and their screening performed by resonance magnetic. ${ }^{28}$

\section{Selective transfection with PEI nanoparticles (polyethylenimine) modified - SSPEI}

Usually, the viral transfection agents are associated with toxicity, limiting their clinical use as vectors. The SSPEI stability in the extracellular environment and the reductive intracellular environment are important compound to the efficacy of transfection process mediated by SSPEI. Also, a new method to produce water-dispersible cellulose carbon nanoparticles-CCN whose had been demonstrated a high cell viability and good efficiency for gene transfection at low concentration. Interestingly, the $\mathrm{CCN}$ was not selectively cytotoxicity even at high doses and their transfection results are comparable to commercial Effectene kit. ${ }^{29}$ However, the polysorbitol mediated transporter-PSMT carrier forms a cover having polysorbitol osmotic property, then when the vehicle binds to the cells and the cells by osmosis through the particles absorb proteins called caveolae. ${ }^{30}$ The transfection detection could be measured by the intensity of cell transgene expression and quantifying the activity of luciferase by a multi-plate luminometer. Transfection activity is expressed as relative light units (RLU) per milligram of cell protein. Furthermore, the level of transfection in cancer cells and normal cells change due to the specific proteins that are over expressed in cancer cells by transfecting agent which has higher affinity.

Another method mediated by Polyplex transfection is also 
used for effective delivery of episomal vectors in the cytoplasm of primary culture of rat embryo fibroblasts. This method belong carrier out the episomal vectors complexed with transfection reagents as Lipofectamine reagents, for example. In these protocols, the ratio of transfection reagent to the commercially available episomal vectors was varied and the transfection efficiency and cytotoxicity of the complexes were subsequently analyzed using flow cytometry and trypan blue exclusion assay, respectively. Although new studies are needed to improve the yield of transfection, using episomal vectors (basis for gene therapy techniques) which avoid non-specific integration of foreign genes in the chromosomes. ${ }^{31}$ Typically, such assays utilize expression of genes for luciferase fluorescent protein, GFP contained in the plasmid, with the time and dose rates for transfection of DNA. HepG2 cells, CHO, NIH 3T3 cells were transfected with PEI and DNA. ${ }^{32}$ The results demonstrated a highly optimized and efficient assay to screen large libraries for gene transfer in vitro.

Nevertheless, selective transfection using an antibody, also based non-viral vector belong to verify protein over expression or knockdown of individual proteins in specific cell populations, especially neuronal cells and investigate cell function in vivo. The knowledge about the DNA transfection the first report of a non-viral vector to selectively transfect tumor cells in the brain, suggesting that cancer cells express different receptors of healthy cells can be a powerful vector interference RNA delivery and other drugs of interest. ${ }^{33}$

\section{Conclusion}

Reinforced the hypothesis of a few changes in the transfection protocols and methods when comparing the old transfection with current methods. Also, new molecules and molecular structures, molecules were developed from conventional structures. Furthermore, new transfection techniques still need to be improved to increase performance in delivering genes as potential therapies for various diseases aiming the gene therapy.

\section{Acknowledgements}

None.

\section{Conflict of interest}

The author declares no conflict of interest.

\section{References}

1. Ausubel FM, Brent R, Kingston RE, et al. Current protocols in molecular biology. Current Protocols in Molecular Biology. 2003. p. 1-4410.

2. Walker JM. Methods in Molecular Biology. Switzerland: A product of Humana Press; 2009.

3. Kwon. Methods in Molecular Biology. Switzerland: A product of Humana Press; 2011.733 p.

4. Baym M, Stone LK, Kishony R. Multidrug evolutionary strategies to reverse antibiotic resistance. Science. 2016;351(6268):aad3292.

5. Vorburger SA, Hunt KK. Adenoviral gene therapy. The Oncologist. 2002;7:46-59.

6. Barquinero J, Eixarch H, Melgosa MP. Retroviral vectors: new applications for an old tool. Gene Ther. 2004;11(Suppl 1):S3-S9.

7. Deichmann A, Schmidt M. Biosafety considerations using gamma-retroviral vectors in gene therapy. C Curr Gene Ther. 2013;13(6):469-477.
8. Danos O. Gene therapy protocols. British journal of cancer. 2009;100(6):1018-1019.

9. Anderson WF. Human gene therapy. Nature. 1998;392:25-30.

10. Pfeifer a, Verma IM. Gene therapy: promises and problems. Annual review of genomics and human genetics. 2001;2:177-211.

11. Soofiyani SR, Baradaran B, Lotfipour F, et al. Gene therapy, early promises, subsequent problems, and recent breakthroughs. Advanced Pharmaceutical Bulletin. 2013;3(2):249-255.

12. Kawai S, Nishizawa M. New procedure for DNA transfection with polycation and dimethyl sulfoxide. Molecular and cellular biology. 1984;4(6):1172-1174.

13. Godbey WT, Barry MA, Saggau P, et al. Poly (ethylenimine)-mediated transfection: A new paradigm for gene delivery. Journal of Biomedical Materials Research. 2000;51(3):321-328.

14. Lin X, Zhao N, Yan P, et al. The shape and size effects of polycation functionalized silica nanoparticles on gene transfection. Acta Biomater. 2000;11:381-392.

15. Cho YW, Kim J, Park K. Polycation gene delivery systems : escape from endosomes to cytosol. Journal of Pharmacy and Pharmacology. 2003;55(6):721-734.

16. Rodriguez RA, Polston PM, Wu MJ, et al. An improved infectivity assay combining cell culture with real-time PCR for rapid quantification of human adenoviruses 41 and semi-quantification of human adenovirus in sewage. Water Res. 2013;47:3183-3191.

17. Graham FL, Van der Eb AJ. A new technique for the assay of infectivity of human adenovirus 5 DNA. Virology. 1973;52(2):456-467.

18. Loyter a, Scangos G a, Ruddle FH. Mechanisms of DNA uptake by mammalian cells: fate of exogenously added DNA monitored by the use of fluorescent dyes. Proc Natl Acad Sci USA. 1982;79(2):422-426.

19. Hawe A, Sutter M, Jiskoot W. Extrinsic fluorescent dyes as tools for protein characterization. Pharm Res. 2008;25(7):1487-1499.

20. Felgner PL. Particulate systems and polymers for in vitro and in vivo delivery of polynucleotides. Advanced Drug Delivery Reviews. 1990;5:163-187.

21. Yamanaka M, Yasuda T. Qcm detection of membrane protein-ligand interactions using cell-derived liposomes. Rsc Org. 2012:1-3.

22. Li P, Liu D, Sun X, et al. A novel cationic liposome formulation for efficient gene delivery via a pulmonary route. Nanotechnology. 2011;22(24):245104.

23. Liu C, Dalby B, Chen W, et al. Transient transfection factors for high-level recombinant protein production in suspension cultured mammalian cells. In Molecular Biotechnology. 2008;39(2):141-153.

24. Srinivasan K, Ramarao P. Animal models in type 2 diabetes research: an overview. Indian J Med Res. 2007;125(3):451-472.

25. Kines KJ, Rinaldi G, Okatcha TI, et al. Electroporation facilitates introduction of reporter transgenes and virions into schistosome eggs. PLoS Neglected Tropical Diseases. 2010;4(2):e593.

26. Metzker ML. Sequencing technologies the next generation. Nat Rev Genet. 2010;11(1):31-46.

27. Han NR, Lee H, Baek S, et al. Delivery of episomal vectors into primary cells by means of commercial transfection reagents. Biochem Biophys Res Commun. 2015;461(2):348-353.

28. Park W, Yang HN, Ling D, et al. Multi-modal transfection agent based on monodisperse magnetic nanoparticles for stem cell gene delivery and tracking. Biomaterials. 2014;35(25):7239-7247. 
29. Zanin H, Hollanda LM, Ceragioli HJ, et al. Carbon nanoparticles for gene transfection in eukaryotic cell lines. Mater Sci Eng C Mater Biol Appl. 2014;39:359-370.

30. Nguyen KCT, Muthiah M, Islam MA, et al. Selective transfection with osmotically active sorbitol modified PEI nanoparticles for enhanced anti-cancer gene therapy. Colloids Surf B Biointerfaces. 2014;119:126136.

31. Donato MT, Lahoz a, Castell JV, et al. Cell lines: a tool for in vitro drug metabolism studies. Curr Drug Metab. 2008;9(1):1-11.
32. Li J, Crowley ST, Duskey J, et al. Miniaturization of gene transfection assays in 384- and 1536-well microplates. Anal Biochem. 2014;470:14 21.

33. Burkholder JK, Decker J, Sun Yang N. Rapid transgene expression in lymphocyte and macrophage primary cultures after particle bombardment-mediated gene transfer. J Immunol Methods. 1993;165(2):149156. 